\title{
Percutaneous endoscopic transforaminal discectomy for the treatment of L5-S1 lumbar disc herniation and the influence of iliac crest height on its clinical effects
}

\author{
QI-CHUN SONG ${ }^{1}$, YAN ZHAO $^{1}$, DONG LI $^{1}$, YUAN-KAI ZHANG ${ }^{1}$, \\ LI-HONG FAN $^{1}$, QUAN-YI LI ${ }^{2}$, DA-PENG DUAN ${ }^{2}$ and ZHI-BIN SHI ${ }^{1}$ \\ ${ }^{1}$ Department of Orthopaedics, The Second Affiliated Hospital of Xi'an Jiaotong University, Xi'an, Shaanxi 710004; \\ ${ }^{2}$ Department of Orthopaedics, The Third Affiliated Hospital of Xi'an Jiaotong University, Xi'an, Shaanxi 710068, P.R. China
}

Received June 26, 2020; Accepted January 22, 2021

DOI: $10.3892 /$ etm.2021.10298

\begin{abstract}
The present study aimed to explore the clinical effects of percutaneous endoscopic transforaminal discectomy using a transforaminal endoscopic spine system (TESSYS) technique for the treatment of L5-S1 lumbar disc herniation and to analyse the influence of iliac crest height on these clinical effects. The clinical data of 76 patients with L5-S1 single-segment disc herniation treated with TESSYS at The Second Affiliated Hospital and Third Affiliated Hospital of Xi'an Jiaotong University between January and December 2016 were retrospectively analysed. Patients were divided into the following three groups according to the positional relation between the highest point of the iliac crest and the L4 and L5 pedicles in the lateral lumbar, as determined by X-ray: Group I, iliac crest height below the upper edge horizontal line of the L5 pedicle $(n=42)$; group II, iliac crest height between the lower edge horizontal line of the L4 pedicle and the upper edge horizontal line of the L5 pedicle $(n=29)$ and group III, iliac crest height above the lower edge horizontal line of the L4 pedicle $(n=5)$. Changes in the postoperative visual analogue scale (VAS) pain score and Oswestry disability index (ODI) of the lower back and lower limbs were observed, and the effects were compared among the three groups. The mean operating time was $86.5 \pm 13.5 \mathrm{~min}$. A single patient experienced cerebrospinal fluid leakage due to a mild tear of the dura mater during the operation, which improved after symptomatic
\end{abstract}

Correspondence to: Professor Quan-Yi Li, Department of Orthopaedics, The Third Affiliated Hospital of Xi'an Jiaotong University, 256 Youyi Road West, Xi'an, Shaanxi 710068, P.R. China E-mail: licool001@163.com

Professor Zhi-Bin Shi, Department of Orthopaedics, The Second Affiliated Hospital of Xi'an Jiaotong University, 157 Xiwu Road, Xi'an, Shaanxi 710004, P.R. China

E-mail: zhibin_shi@126.com

Key words: PETD, lumbar disc herniation, L5-S1, crest height, minimally invasive spine treatment. The same operation was repeated in one patient due to the recurrence of disc herniation. In all patients, the VAS pain score and ODI of the lower back and lower limbs at 1 week and 1,3 and 12 months following the operation were significantly lower than those before the operation (all $\mathrm{P}<0.05$ ). Furthermore, the postoperative VAS pain score and ODI of the lower back and lower limbs were poorer in group III (L5-S1 lumbar disc herniation complicated with high iliac crest) than in groups I and II $(\mathrm{P}<0.05)$. These results suggested that TESSYS was effective in treating lumbar disc herniation. Whether the iliac crest is higher than the lower edge horizontal line of the L4 pedicle is suggested to be one of the factors influencing the outcome of the operation.

\section{Introduction}

Continuous refinement of percutaneous endoscopic transforaminal discectomy (PETD) has resulted in the development of an effective treatment for intraspinal herniation, intervertebral foramen herniation, polar lateral herniation and intervertebral disc prolapse (1). With the invention and use of new tools, including the ring saw and the microscopical abrasion drill, PETD has evolved from indirect decompression, by removing only the central nucleus pulposus, to direct decompression, by removing the intervertebral disc (2). PETD is widely used owing to its many advantages, including minimal operative wound, minor effect on spinal stability, reduced postoperative pain and local scar formation and fast recovery (3). However, operation at the L5-S1 segment is more difficult, owing to the occlusion of the iliac crest, inclination of the intervertebral disc, stenosis of the intervertebral foramen and the presence of other complex anatomical structures (4).

Operation at the L5-S1 segment is challenging given the anatomical structure of this region. A number of scholars believe that the working casing cannot increase the angle with the centre line from the horizontal plane of the L5-S1 segment; therefore, it is impossible to overcome the occlusion of the herniated disc by the facet joint (4). From the coronal point of view, the working casing is not in the same horizontal plane as the intervertebral space, making it more difficult to remove the disc tissue. Lee et al (5) suggest that the higher iliac crest and tilted L5-S1 intervertebral disc increase the 
angle of the working casing and enable deviation from the intervertebral disc and that whether the casing is in a good position directly affects the success or failure of the operation. Ahn et al (6) believe that the special anatomical structure of the L5-S1 segment often leads to prolonged operation time, increased radiation exposure and even operation failure. A study has proposed moving the iliac crest downwards by through changing the patient's position may be beneficial, however, this operation still has many difficulties in its practical applications (7).

To overcome the challenging anatomical factors, percutaneous endoscopic interlaminar discectomy (PEID) is also a common approach for operation at the L5-S1 segment. This method can effectively avoid occlusion of the iliac crest (8). The most significant difference between PETD and PEID is that PEID exposes the nerve tissue and then treats the disc, whereas PTED treats the disc first and finally exposes the nerve tissue. PEID causes greater stimulation of the nervous tissue, and patients under local anaesthesia have poor intraoperative tolerance and are more likely to have nerve injuries (9). The endoscopic transiliac approach proposed by Choi et al (10) can reduce the working channel inclination and direct it to the L5-S1 intervertebral foramen, lowering the possibility of damaging the exiting root nerve. However, using this approach results in the casing developing a narrow range of movement after the establishment of the working channel, possibly resulting in ilium fractures, gluteal epithelial nerve damage and uncontrollable bleeding $(4,8,9)$. These factors have limited the development of the endoscopic transiliac approach.

Two percutaneous endoscopic operations, namely endoscopic discectomy via the intervertebral foramen approach and endoscopic discectomy via the posterior interlaminar approach, are currently available for L5-S1 lumbar disc herniation; however, both these approaches have advantages and disadvantages. Previous studies have demonstrated that PETD is preferred for shoulder type recurrent disc herniation that is centrally located, while PEID is preferred for axillary type migrated discs, especially those of a high grade $(8,9)$. Numerous studies have proposed that a high iliac crest is the major obstacle for PETD at the L5-S1 segment or even the L4-L5 segment $(4,9,11,12)$. However, a 'high iliac crest' itself is a vague definition without quantitative standards. Furthermore, a study on the influence of the iliac crest on L5-S1 segment surgery have rarely been reported (4).

In the present study, the TESSYS technique in PTED invented by Hoogland (13) was used to treat 76 patients with L5-S1 lumbar disc herniation. Furthermore, the clinical effects of this technique and the influence of the iliac crest height on the clinical effects were analysed.

\section{Materials and methods}

Inclusion criteria. The inclusion criteria were as follows: i) No lumbar instability at the L5-S1 segments was shown on the $\mathrm{X}$-ray films taken in the lumbar dynamic position; ii) L5-S1 single-segment lumbar disc herniation; iii) no complications of spinal diseases, including lumbar spinal stenosis, spinal deformity and spinal tuberculosis and iv) ineffective conservative treatment. A total of 76 patients with lumbar disc herniation, including 46 male and 30 female patients aged 19-69 (mean
A

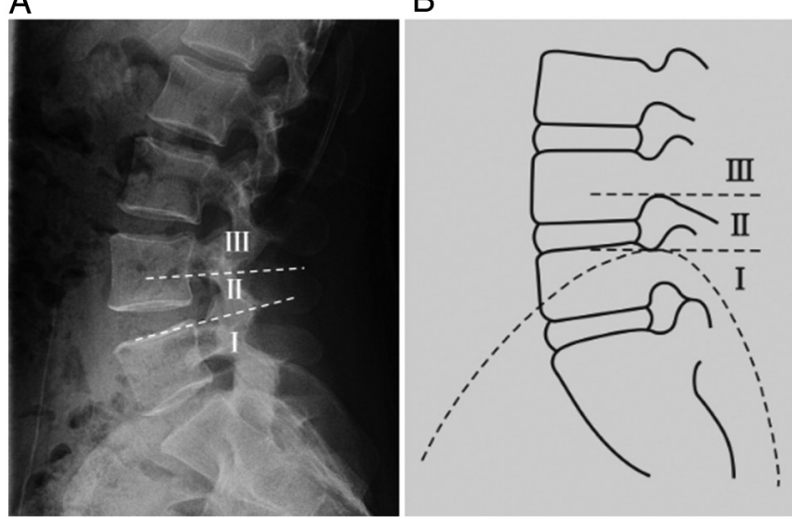

Figure 1. On the basis of the relationship between the highest point of the iliac crest and the L4 and L5 pedicles on the lumbar lateral radiograph, patients were divided into three groups. (A) Lateral radiography of the lumbar and (B) corresponding schematic illustration. The dotted line above represents the lower edge horizontal line of the L4 pedicle and the dotted line below demonstrates the upper edge horizontal line of the L5 pedicle. I represents iliac crest height below the upper edge horizontal line of the L5 pedicle. II indicates the iliac crest height between the lower edge horizontal line of the L4 pedicle and the upper edge horizontal line of the L5 pedicle. III represents the iliac crest height above the lower edge horizontal line of the L4 pedicle.

age, 47.8 \pm 6.4 ) years, met the inclusion criteria and were treated with the THESSYS technique at the Second Affiliated Hospital and Third Affiliated Hospital of Xi'an Jiaotong University between January and December 2016. The study was approved by the Medical and Biological Ethics Committee of Xi'an Jiaotong University Health Science Center, and all participants signed the informed consent to the clinical study. These patients were divided into the following three groups according to the positional relation between the highest point of the iliac crest and the L4 and L5 pedicles in the lateral lumbar X-ray film: Group I, iliac crest height below the upper edge horizontal line of the L5 pedicle $(n=42)$; group II, with iliac crest height between the lower edge horizontal line of the L4 pedicle and the upper edge horizontal line of the L5 pedicle $(n=29)$ and group III, iliac crest height above the lower edge horizontal line of the L4 pedicle $(n=5)$ (Fig. 1).

Operation methods. Patients were placed on their healthy side (or on the side with mild symptoms if the patient has symptoms on both sides). The puncture point was determined via anteroposter fluoroscopy (10-12 cm in the midline after paraperitoneal opening and $1 \mathrm{~cm}$ above the iliac crest). Then, $1 \%$ lidocaine was used for local anaesthesia. A sharp knife was used to place the guide wire, guide rod, casing and ring saw step by step at a site approximately $0.8 \mathrm{~cm}$ from the puncture point incision. Finally, a $7.5 \mathrm{~cm}$ working casing was placed. The intervertebral foramen mirror was connected to the light source and camera when the fluoroscope shows that the normal position of the tip of the working casing is on the inner edge of the vertebral pedicle and laterally on the posterior edge of the vertebral body (TESSYS technique). The degenerated and herniated disc tissues were completely removed using flexible nucleus pulposus forceps by rotating the working channel during the operation, followed by radio frequency haemostasis and ablation. The criteria for ending the operation were S1 nerve root relaxation after decompression and normal 
Table I. Comparison of the general data of patients with disc herniation among the three groups.

\begin{tabular}{|c|c|c|c|c|c|c|c|}
\hline \multirow[b]{2}{*}{ Group } & \multirow{2}{*}{$\begin{array}{l}\text { Number } \\
\text { of cases }\end{array}$} & \multirow[b]{2}{*}{ Age/years } & \multicolumn{2}{|c|}{ Sex/number of cases } & \multirow[b]{2}{*}{ Course/month } & \multirow[b]{2}{*}{ VAS score/points } & \multirow[b]{2}{*}{ ODI/points } \\
\hline & & & Male & Female & & & \\
\hline Group I & 42 & $46.9 \pm 7.2$ & 19 & 23 & $12.3 \pm 2.6$ & $7.8 \pm 1.2$ & $52.1 \pm 9.0$ \\
\hline Group II & 29 & $48.3 \pm 6.6$ & 13 & 16 & $11.2 \pm 3.1$ & $7.7 \pm 1.3$ & $53.0 \pm 8.1$ \\
\hline Group III & 5 & $47.7 \pm 8.2$ & 2 & 3 & $13.0 \pm 2.9$ & $7.9 \pm 2.5$ & $55.9 \pm 9.9$ \\
\hline Statistical tests & & $\mathrm{F}=0.342$ & $\chi^{2}=0.050$ & & $F=1.695$ & $\mathrm{~F}=0.073$ & $\mathrm{~F}=0.339$ \\
\hline P-value & & 0.712 & 0.957 & & 0.191 & 0.929 & 0.714 \\
\hline
\end{tabular}

Comparison between all groups was performed with One-Way ANOVA and Tukey's tests. Comparison of sex between three groups was examined using the $\chi^{2}$ test. ODI, Oswestry disability index; VAS, visual analogue scale.

Table II. Comparison of VAS pain score and ODI of patients with disc herniation before and after the operation (mean \pm SD, points).

\begin{tabular}{|c|c|c|c|c|c|c|c|}
\hline Parameter & $\begin{array}{l}\text { Before } \\
\text { OP }\end{array}$ & $\begin{array}{c}1 \text { week } \\
\text { after OP }\end{array}$ & $\begin{array}{l}1 \text { month } \\
\text { after OP }\end{array}$ & $\begin{array}{l}3 \text { months } \\
\text { after OP }\end{array}$ & $\begin{array}{l}12 \text { months } \\
\text { after OP }\end{array}$ & F value & P-value \\
\hline $\begin{array}{l}\text { VAS score of lower back } \\
(n=61)\end{array}$ & $7.9 \pm 1.1$ & $3.6 \pm 1.3^{\mathrm{a}}$ & $2.4 \pm 0.9^{\mathrm{a}}$ & $1.3 \pm 1.0^{\mathrm{a}}$ & $1.5 \pm 1.3^{\mathrm{a}}$ & 434.80 & 0.001 \\
\hline $\begin{array}{l}\text { VAS score of lower limbs } \\
(n=68)\end{array}$ & $7.7 \pm 1.2$ & $3.7 \pm 1.4^{\mathrm{a}}$ & $1.7 \pm 1.4^{\mathrm{a}}$ & $1.7 \pm 1.1^{\mathrm{a}}$ & $1.8 \pm 1.2^{\mathrm{a}}$ & 318.90 & 0.001 \\
\hline $\begin{array}{l}\text { ODI } \\
(n=76)\end{array}$ & $52.3 \pm 9.6$ & $11.6 \pm 7.8^{\mathrm{a}}$ & $10.9 \pm 8^{\mathrm{a}}$ & $9.2 \pm 7.9^{\mathrm{a}}$ & $10.0 \pm 9.0^{\mathrm{a}}$ & 367.30 & 0.001 \\
\hline
\end{tabular}

Comparison between all groups was performed with One-Way ANOVA and Tukey's tests. ${ }^{\mathrm{a}} \mathrm{P}<0.05$ vs. before OP. ODI, Oswestry disability index; VAS, visual analogue scale; OP, operation.
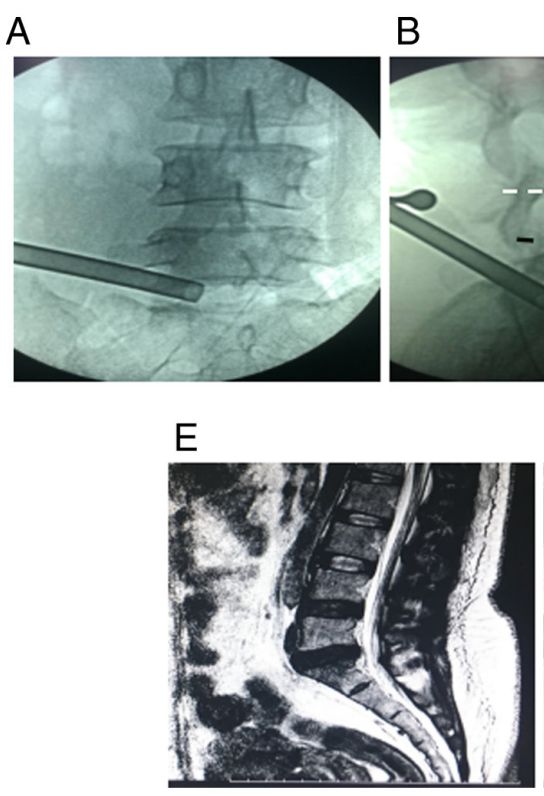

$\mathrm{F}$

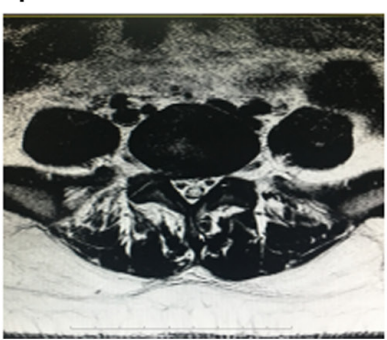

G

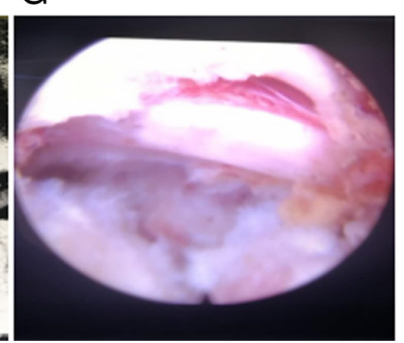

Figure 2. Photographs before and after PETD for L5-S1 lumbar intervertebral disc herniation in a 45-year-old woman. (A) Intraoperative images of the working cannula in anteroposterior radiography of the lumbar. (B) Intraoperative images of the working cannula in lateral radiography of the lumbar. The upper edge of the L5 pedicle is shown in black, and the iliac crest is slightly below this line in this case. (C) Preoperative sagittal section of lumbar magnetic resonance imaging. (D) Preoperative transverse section of lumbar magnetic resonance imaging showing right-sided subaxillary herniation at the L5-S1 segments and a large herniated disc that compresses the dural sac and the right S1 nerve root. (E) Postoperative sagittal section of lumbar magnetic resonance imaging at 1 month after the operation. (F) Postoperative transverse section of lumbar magnetic resonance imaging at 1 month after the operation showing that the herniated L5-S1 disc has disappeared and that the dural sac and the S1 nerve root compression on the right side has been relieved. (G) The decompressed nerve roots can be seen after complete removal of the herniated disc tissue. 
pulsation of the dural sac area or collapse of the posterior longitudinal ligament.

Evaluation indicators. The duration of each operation was recorded, perioperative complications were observed and the Oswestry Disability Index (ODI) (14) and Visual Analogue Scale (VAS) (15) of the lower back and lower limbs before operation and 1 week and 1,3 and 12 months after the operation were evaluated. Higher VAS scores indicate more severe pain, and higher ODIs indicate more serious dysfunction.

Statistical analysis. The statistical software SPSS 18.0 (IBM Corp.) was used for data analysis. Data are presented as the mean \pm SD. Repeated measures analysis of variance was used for comparisons before and after the operation, followed by a Tukey's post hoc test for comparisons among groups. One-Way ANOVA was performed for the comparisons between three groups, and the Tukey's post hoc test was used for the comparisons among groups. The differences of sex between three groups were examined using the $\chi^{2}$ test. $\mathrm{P}<0.05$ was considered statistically significant.

\section{Results}

Baseline data of patients. Differences in sex, age, disease course, VAS, ODI and other general data among the three groups were not statistically significant (all $\mathrm{P}>0.05$ ). These data were therefore comparable (Table I).

Comparison of VAS and ODI scores before and after the operation. All 76 patients successfully underwent surgery with a mean operating time of $86.5 \pm 13.5 \mathrm{~min}$ and a mean intraoperative haemorrhage of $23.01 \pm 1.45 \mathrm{ml}$. One patient had cerebrospinal fluid leakage due to a mild tear of the dura mater during the operation, which improved after symptomatic treatment. The same operation was repeated in one patient due to recurrence of disc herniation, after which the patient's condition improved. The follow-up period was 12-17 (mean 12.4 \pm 2.4 ) months. No complications, including epidural haematoma, incision infection, lumbar instability and refractory low back pain, occurred during the follow-up period. Among the 76 patients followed-up, 61 had low back pain and 68 had radiating pain in the lower limbs before the operation. As shown in Table II, the VAS scores and ODI of the lower back and lower limbs were significantly improved in both groups after the operation $(\mathrm{P}<0.05)$.

Comparison of intraoperative conditions and postoperative clinical effects between the three groups. As shown in Table III, the differences in the duration of operation, amount of intraoperative bleeding and the VAS score and ODI of the lower back and lower limbs at each time point after the operation between group III and groups I and II were statistically significant $(\mathrm{P}<0.05)$, while those between group I and group II were not statistically significant $(\mathrm{P}>0.05)$. Intraoperative complications occurred in two patients with high iliac crest in group III. One had cerebrospinal fluid leakage due to a mild tear of the dura mater during the operation, which improved after symptomatic treatment. The same operation was repeated in another patient due to recurrence

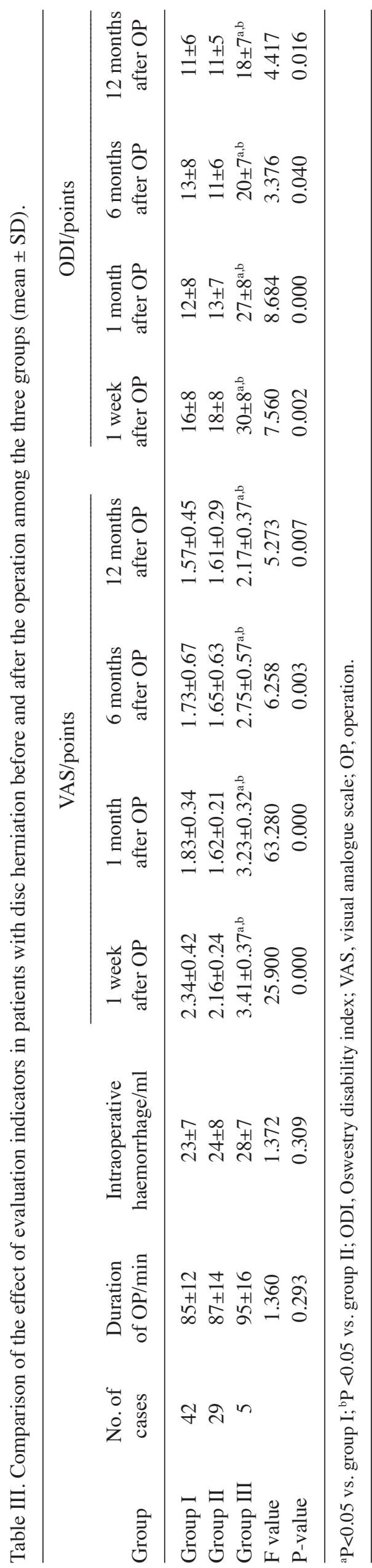


of disc herniation, after which the condition of this patient improved (Fig. 2).

\section{Discussion}

Advantages and effects of the TESSYS technique on the treatment of lumbar disc herniation. In 2002, Hoogland et al (13) proposed the TESSYS technique on the basis of the Yenug Endoscopic Spine System technique, resulting in advances in PETD. The TESSYS technique points the puncture target to the posterior edge of the vertebral body, adding a special ring saw at the same time to partially excise the bony structure of the intervertebral foramen, and enters the spinal canal through the enlarged intervertebral foramen. These steps achieve direct decompression and spinal canal decompression, broaden the indications and improve the treatment level of lumbar disc herniation. At present, this technique can treat almost all types of lumbar disc herniation (including giant, prolapsed and revision operation) (16). The technique is also suitable for treating lumbar spinal stenosis, nerve root canal stenosis and other diseases $(17,18)$.

Numerous studies have shown that the treatment of lumbar disc herniation using PETD can achieve the same effect observed using traditional open operation or microendoscopic discectomy (19-22). Furthermore, this technique is a safe and effective minimally invasive method. The results of this study also showed only small amounts of intraoperative bleeding, significantly improved VAS pain scores and ODI of the lower back and the lower limbs at each time point and low incidence of intraoperative complications, which is similar to previous studies (21-23).

Patients' iliac crest height and operative approaches. For patients with L5-S1 disc herniation, the puncture angle of the intervertebral foramen approach to the herniated nucleus pulposus is steep, due to the presence of anatomical factors, such as high iliac crest, large zygopophysis at S1 and large inclination of the L5-S1 intervertebral space, resulting in certain difficulties during operation. Choi et al (9) have suggested that the highest point of the iliac crest above the L5 pedicle will make the operation via the intervertebral foramen approach less effective. Because the L5-S1 interlaminar space is relatively wide, scholars have also suggested solving disc herniation caused by high iliac crest via an interlaminar approach (24). Ruetten et al (25) reported that the interlaminar approach was selected in $94.7 \%$ of the patients with L5-S1 disc herniation and that the operative effects were excellent. However, other scholars suggest that for L5-S1 intervertebral disc herniation with upper and lower migration, the intervertebral foramen or intervertebral lamina approach drilled through the ilium may be more effective $(26,27)$. The results of the present study suggest that during the excision of the nucleus pulposus by the interlaminar approach for the treatment of L5-S1 intervertebral disc herniation, the effect of local anaesthesia was poor, and the average VAS pain score was high. Therefore, the majority of patients continued the operation after optimizing anaesthesia with general anaesthesia. At present, the operation is usually performed through an interlaminar approach under general anaesthesia to avoid failure procedure $(28,29)$, To encourage cooperation of patients under local anaesthesia, the intervertebral foramen approach was still adopted for patients with high iliac crest in the current study.

Influence of patients' iliac crest height on the operative effect of the TESSYS technique. A high iliac crest is a major obstacle to percutaneous endoscopic lumbar discectomy at the L5-S1 segment $(4,24)$. However, a 'high iliac crest' itself is a vague definition without quantitative standards. In the present study, the height of the iliac crest above the horizontal line of the lower edge of the L4 pedicle demonstrated a significant influence on the postoperative effect of TESSYS. The postoperative ODI and VAS scores of the lower back and lower limbs in group III were much lower than that those in groups I and II, and the degree of pain relief and functional recovery of the lower back and lower limbs after operation in group III was also lower than those in groups I and II; one patient with recurrence and one with dural tear were also from the high iliac crest group. Choi and Park (4) and Choi et al (9) suggest that, in lateral PETD, iliac crest height has different effects on different types of herniation in the treatment of L5-S1 intervertebral disc herniation and is more effective for shoulder and lateral herniations; however, the present study did not perform group comparison based on types of herniations. The current study revealed that the operative space was limited, as a lateral approach would be seriously obstructed by the L5 transverse process or one sacral transverse process relic in case of high iliac crest; therefore, it is difficult to adjust the working casing during the operation.

In practice, the operation can usually be performed successfully when iliac crest height is below the upper edge horizontal line of the L5 pedicle in the present study. In addition, the current study revealed that the operation can be completed by experienced operators when iliac crest height is between the lower edge horizontal line of the L4 pedicle and the upper edge horizontal line of the L5 pedicle. Moreover, alternative approaches are recommended when iliac crest height is above the lower edge horizontal line of the L4 pedicle. The operative position also has a certain effect on the puncture angle. In Choi et al (9) study patients were placed in a prone position. However, in the present study, patients were placed in a lateral position with a cushion under the lower back to open the intervertebral foramen to a certain extent. In such a case, the upper edge of the ilium has a certain downward movement relative to the level of L5-S1, which is conducive for the removal of the prominent nucleus pulposus, which may be the main reason for the good effects in group II in this study.

For L5-S1 disc herniation with a high iliac crest, although the angle of the transforaminal puncture is high, intervertebral foramen formation and the lateral position of the patients can broaden the operative field. Therefore, the target is easily reached and the range of decompression increases $(4,30)$. As a result, even for patients with high iliac crest, the VAS score and ODI were significantly improved after the operation, fully indicating the exact effect of the TESSYS technique. In the present study, VAS score and ODI of patients showed an increase at 1 year postoperatively, but there was no significant difference, which may be due to application of drugs to relieve pain at an early stage, tiredness after returning to work in the later stage, or it may be caused by the small sample size. To 
avoid spinal instability, the superior articular process was ground as much as was possible to ensure that the range of resection of the S1 superior articular process was less when the intervertebral foramen was formed. Therefore, no spinal instability was observed during the 1-year follow-up visit.

In the present study, only one patient had cerebrospinal fluid leakage and one had postoperative recurrence, which is consistent with the incidence of complications reported in the literature (24). Both complications occurred in patients with an iliac crest height above the lower edge horizontal line of the L4 pedicle. For patients with high iliac crest, operation should be performed carefully. Measures such as complete decompression, delaying postoperative off-bed activities, or expansion of the intervertebral foramen and selection of the intervertebral lamina approach as appropriate should be considered to reduce the incidence of complications.

The relationship between the iliac crest and L4 and L5 pedicles in the lumbar lateral X-ray film was used to grade iliac crest height, which effectively avoided individual anatomical differences, such as sex, height and transitional vertebrae. It objectively reflected the influence of the iliac crest on PETD, which was convenient for the operator to evaluate the operation difficulty and had an effective guiding role in the selection of the surgical approach. However, one limitation of the present study is that only the iliac wing height, not the wing area, was considered, as the occlusion of the iliac wing margin from front to back may also have a great impact on the operation of the L5-S1 segment. In addition, the sample size of group III in this study was small, which may have biased the results. Therefore, future studies will require an increased sample size and assess the influence of the iliac wing on the operation to obtain more convincing conclusions.

The present data suggest that the TESSYS technique is effective in treating lumbar disc herniation. Whether the iliac crest is higher than the lower edge horizontal line of the L4 pedicle is one of the factors influencing the operative outcome.

\section{Acknowledgements}

Not applicable.

\section{Funding}

The present study was funded by the National Natural Science Foundation of China, (grant no. 82002311).

\section{Availability of data and materials}

The datasets used and/or analyzed during the current study are available from the corresponding author on reasonable request.

\section{Authors' contributions}

QCS, ZBS and QYL contributed to the conception and design. YZ, DL, QYL and LHF performed the experiments. YKZ and DPD contributed to data acquisition and analysis. QCS and YZ wrote the manuscript. QCS and ZBS confirmed the authenticity of all the raw data. All authors have read and approved the final manuscript.

\section{Ethics approval and consent to participate}

The study was approved by the Medical and Biological Ethics Committee of Xi'an Jiaotong University Health Science Center and all patients provided their written informed consent.

\section{Patient consent for publication}

Not applicable.

\section{Competing interests}

The authors declare that they have no competing interests.

\section{References}

1. Choi G, Pophale CS, Patel B and Uniyal P: Endoscopic spine surgery. J Korean Neurosurg Soc 60: 485-497, 2017.

2. Ahn Y, Lee SH, Park WM, Lee HY, Shin SW and Kang HY: Percutaneous endoscopic lumbar discectomy for recurrent disc herniation: Surgical technique, outcome, and prognostic factors of 43 consecutive cases. Spine (Phila Pa 1976) 29: E326-E332, 2004.

3. Sairyo K, Egawa H, Matsuura T, Takahashi M, Higashino K, Sakai T, Suzue N, Hamada D, Goto T, Takata Y, et al: State of the art: Transforaminal approach for percutaneous endoscopic lumbar discectomy under local anesthesia. J Med Invest 61: 217-225, 2014.

4. Choi KC and Park CK: Percutaneous endoscopic lumbar discectomy for L5-S1 disc herniation: Consideration of the relation between the iliac crest and L5-S1 disc. Pain Phys 19: E301-E308, 2016.

5. Lee SH, Choi KC, Baek OK, Kim HJ and Yoo SH: Percutaneous endoscopic intra-annular subligamentous herniotomy for large central disc herniation: A technical case report. Spine (Phila Pa 1976) 39: E473-E479, 2014.

6. Ahn Y, Kim CH, Lee JH, Lee SH and Kim JS: Radiation exposure to the surgeon during percutaneous endoscopic lumbar discectomy: A prospective study. Spine (Phila Pa 1976) 38: 617-625, 2013.

7. Ahn Y: Transforaminal percutaneous endoscopic lumbar discectomy: Technical tips to prevent complications. Expert Rev Med Devices 9: 361-366, 2012.

8. Nie H, Zeng J, Song Y, Chen G, Wang X, Li Z, Jiang H and Kong Q: Percutaneous endoscopic lumbar discectomy for L5-S1 disc herniation via an interlaminar approach versus a transforaminal approach: A prospective randomized controlled study with 2-year follow up. Spine (Phila Pa 1976) 41 (Suppl 19): B30-B37, 2016.

9. Choi KC, Kim JS, Ryu KS, Kang BU, Ahn Y and Lee SH: Percutaneous endoscopic lumbar discectomy for L5-S1 disc herniation: Transforaminal versus interlaminar approach. Pain Phys 16: 547-556, 2013.

10. Choi G, Kim JS, Lokhande P and Lee SH: Percutaneous endoscopic lumbar discectomy by transiliac approach: A case report. Spine (Phila Pa 1976) 34: E443-E446, 2009.

11. Eun SS, Lee SH, Liu WC and Erken HY: A novel preoperative trajectory evaluation method for L5-S1 transforaminal percutaneous endoscopic lumbar discectomy. Spine 18: 1286-1291, 2018.

12. Tezuka F, Sakai T, Abe M, Yamashita K, Takata Y, Higashino K, Chikawa T, Nagamachi A and Sairyo K: Anatomical considerations of the iliac crest on percutaneous endoscopic discectomy using a transforaminal approach. Spine J 17: 1875-1880, 2017.

13. Hoogland T, Schubert M, Miklitz B and Ramirez A: Transforaminal posterolateral endoscopic discectomy with or without the combination of a low-dose chymopapain: A prospective randomized study in 280 consecutive cases. Spine (Phila $\mathrm{Pa}$ 1976) 31: E890-E897, 2006

14. Fairbank JC and Pynsent PB: The oswestry disability index. Spine 25: 2940-2952, 2000.

15. Myles PS: The pain visual analog scale: Linear or nonlinear? Anesthesiology 100: 744-745, 2004.

16. Kim HS, Paudel B, Jang JS, Lee K, Oh SH and Jang IT: Percutaneous endoscopic lumbar discectomy for all types of lumbar disc herniations (LDH) including severely difficult and extremely difficult LDH cases. Pain Phys 21: E401-E408, 2018. 
17. Wen $B$, Zhang $X$, Zhang L, Huang $P$ and Zheng G: Percutaneous endoscopic transforaminal lumbar spinal canal decompression for lumbar spinal stenosis. Medicine (Baltimore) 95: e5186, 2016.

18. Ahn Y, Lee SH, Park WM and Lee HY: Posterolateral percutaneous endoscopic lumbar foraminotomy for L5-S1 foraminal or lateral exit zone stenosis. Technical note. J Neurosurg 99 (Suppl 3): S320-S323, 2003.

19. Choi KC, Kim JS and Park CK: Percutaneous endoscopic lumbar discectomy as an alternative to open lumbar microdiscectomy for large lumbar disc herniation. Pain Phys 19: E291-E300, 2016.

20. Liu X, Yuan S, Tian Y, Wang L, Gong L, Zheng Y and Li J: Comparison of percutaneous endoscopic transforaminal discectomy, microendoscopic discectomy, and microdiscectomy for symptomatic lumbar disc herniation: Minimum 2-year follow-up results. J Neurosurg Spine 28: 317-325, 2018.

21. Ahn SS, Kim SH, Kim DW and Lee BH: Comparison of outcomes of percutaneous endoscopic lumbar discectomy and open lumbar microdiscectomy for young adults: A retrospective matched cohort study. World Neurosurg 86: 250-258, 2016.

22. Shi R, Wang F, Hong X, Wang YT, Bao JP, Liu L, Wang XH, Xie ZY and Wu XT: Comparison of percutaneous endoscopic lumbar discectomy versus microendoscopic discectomy for the treatment of lumbar disc herniation: A meta-analysis. Int Orthop 43: 923-937, 2019.

23. Ahn Y: Endoscopic spine discectomy: Indications and outcomes. Int Orthop 43: 909-916, 2019

24. Chen J, Jing X, Li C, Jiang Y, Cheng S and Ma J: Percutaneous endoscopic lumbar discectomy for L5S1 lumbar disc herniation using a transforaminal approach versus an interlaminar approach: A systematic review and meta-analysis. World Neurosurg 116: 412-420.e2, 2018
25. Ruetten S, Komp M, Merk H and Godolias G: Surgical treatment for lumbar lateral recess stenosis with the full-endoscopic interlaminar approach versus conventional microsurgical technique: A prospective, randomized, controlled study. J Neurosurg Spine 10: 476-485, 2009.

26. Patgaonkar P, Datar G, Agrawal U, Palanikumar C, Agrawal A, Goyal V and Patel V: Suprailiac versus transiliac approach in transforaminal endoscopic discectomy at L5-S1: A new surgical classification of L5-iliac crest relationship and guidelines for approach. J Spine Surg 6 (Suppl 1): S145-S154, 2020.

27. Osman SG, Sherlekar S, Malik A, Winters C, Grewal PK, Narayanan $\mathrm{M}$ and Gemechu N: Endoscopic trans-iliac approach to L5-S1 disc and foramen-a report on clinical experience. Int J Spine Surg 8: 20-31, 2014.

28. Hua W, Tu J, Li S, Wu X, Zhang Y, Gao Y, Zeng X, Yang S and Yang C: Full-endoscopic discectomy via the interlaminar approach for disc herniation at L4-L5 and L5-S1: An observational study. Medicine (Baltimore) 97: e0585, 2018.

29. Wang D, Xie W, Cao W, He S, Fan G and Zhang H: A cost-utility analysis of percutaneous endoscopic lumbar discectomy for L5-S1 lumbar disc herniation: Transforaminal versus interlaminar. Spine (Phila Pa 1976) 44: 563-570, 2019.

30. Choi KC, Kim JS, Lee DC and Park CK: Percutaneous endoscopic lumbar discectomy: Minimally invasive technique for multiple episodes of lumbar disc herniation. BMC Musculoskelet Disord 18: 329, 2017.

This work is licensed under a Creative Commons Attribution-NonCommercial-NoDerivatives 4.0 International (CC BY-NC-ND 4.0) License. 